\title{
GENERALIZATION OF THE JENSEN-MERCER
} INEQUALITY BY TAYLOR'S POLYNOMIAL

\begin{abstract}
A. MATKOVIĆ
Abstract. We present generalizations of the Jensen-Mercer inequality for the class of $n$-convex functions, obtained by using Taylor's polynomial and Green function. By applying those inequalities we obtain some results related to Čebyšev functionals.
\end{abstract}

Mathematics subject classification (2010): 26D15, 26D20.

Keywords and phrases: Jensen-Mercer inequality, $n$-convex functions, exponential convexity.

\section{REFERENCES}

[1] J. BARIĆ, A. MATKOVIĆ, Bounds for the normalized Jensen-Mercer functional, J. Math. Inequal. 3 (4) (2009), 529-541.

[2] S. I. Butt, K. A. Khan, J. PeČArić, Popovicu type inequalities via Green function and generalized Montgomery identity, Math. Inequal. Appl. 18 (4) (2015), 1519-1538.

[3] P. Cerone, S. S. Dragomir, Some new Ostrowski-type bounds for the Čebyšev functional and applications, J. Math. Inequal. 8 (1) (2014), 159-170.

[4] J. JAKŠETIĆ, J. PEČARIĆ, Exponential convexity method, J. Convex Anal. 20 (1) (2013), 181-197.

[5] J. JAKŠETIĆ, J. PEČARIĆ, A. PERUŠIĆ, Steffensen inequality, higher order convexity and exponential convexity, Rend. Circ. Mat. Palermo. 63 (1) (2014), 109-127.

[6] M. Adil Khan, N. Latif And J. PeČARIĆ, Generalization of majorization theorem, J. Math. Inequal. 9 (3) (2015), 847-872.

[7] J. E. PeČarić, F. Proschan and Y. L. Tong, Convex Functions, Partial Orderings, and Statistical Applications, Academic Press, Inc. 1992. 